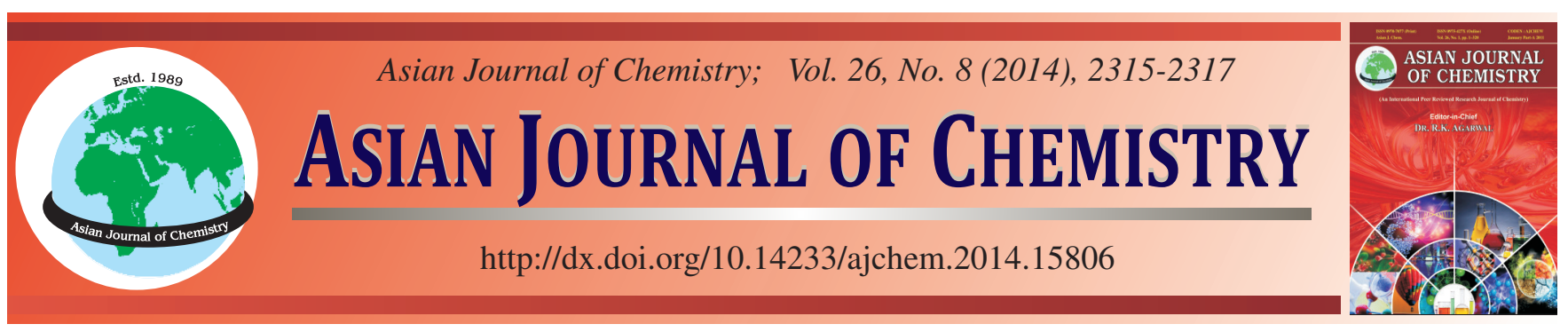

\title{
Simultaneous HPLC Determination of Five Active Ingredients in Belladonna tincture
}

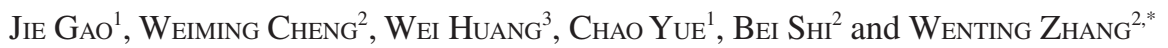

${ }^{1}$ Zhejiang Chinese Medical University, No. 548, Binwen Road, Binjiang Dist. 310053, Hangzhou Zhejiang Province, P.R. China

${ }^{2}$ Zhejiang Institute for Food and Drug Control, No 86 Lane 1, Jichang Road, Jianggan Dist. 310004, Hangzhou Zhejiang Province, P.R. China

${ }^{3}$ Zhejiang Medical College, No. 548 Binwen Road, Binjiang Dist. 310053, Hangzhou Zhejiang Province, P.R. China

*Corresponding author: Tel: +86 13757170891; E-mail: leozhwt@163.com; weiming_cheng@hotmail.com

\begin{abstract}
An HPLC method is established for the simultaneous determination of five active ingredients (scopolin, chlorogenic acid, kaempferol-3O- $\beta$-D (6-O- $\alpha$-L-rhamnogalactoside)-7-O- $\beta$-D-glucoside, scopoletin and rutin). HPLC conditions included Agilent Kromesil 100-5 $\mathrm{C}_{18}$ column $(4.6 \mathrm{~mm} \times 250 \mathrm{~mm}, 5 \mu \mathrm{m})$ and the mobile phase was a mixture of methanol and $0.05 \%$ phosphoric acid for gradient elution. The flow rate was $1 \mathrm{~mL} / \mathrm{min}$. The column temperature was at $30^{\circ} \mathrm{C}$. Five active ingredients had good linear relationships: Scopolin in the range of $0.0326-0.978 \mu \mathrm{g}\left(\mathrm{R}^{2}=0.9999\right)$, chlorogenic acid in the range of $0.0100-0.300 \mu \mathrm{g}\left(\mathrm{R}^{2}=0.9996\right)$, kaempferol-3-O- $\beta-\mathrm{D}(6-\mathrm{O}-\alpha-\mathrm{L}-$ rhamnogalactoside)-7-O- $\beta$-D-glucoside of $0.0319-0.957 \mu \mathrm{g}\left(\mathrm{R}^{2}=0.9996\right)$, scopoletin in the range of $0.0363-1.089 \mu \mathrm{g}\left(\mathrm{R}^{2}=0.9995\right)$ and rutin in the range of $0.0101-0.303 \mu \mathrm{g}\left(\mathrm{R}^{2}=0.9994\right)$. Their average recoveries were $99.59 \%(\mathrm{RSD}=1.9 \%), 96.92 \%(\mathrm{RSD}=1.9 \%)$, $96.41 \%(\mathrm{RSD}=1.5 \%), 96.46 \%(\mathrm{RSD}=2.2 \%)$ and $97.45 \%(\mathrm{RSD}=1.9 \%)$, respectively. This method was steady with high precision and good repeatability. It could be used for the determination of the five active ingredients and quality control of Belladonna tincture.
\end{abstract}

Keywords: HPLC, Belladonna tincture, Chlorogenic acid, Kaempferol-3-O- $\beta$-D(6-O- $\alpha$-L-rhamnogalactoside)-7-O- $\beta$-D-glucoside. ᄂ _ - - - - - - - - - - - - - - - - - - - - - - - - - - -

\section{INTRODUCTION}

Belladonna tincture (B. tincture) is one brownish red or green single tincture processed by Belladonna herb, whose active compounds are mainly tropane alkaloids and flavones, being used for treating gastric and duodenal ulcer, smooth muscle spasm, inhibition of glandular secretion, angina of gastrointestinal tract, biliary cholic, renal cholic, abdominal pain caused by ureteral calculus, vomiting and diarrhea due to gastritis or gastrospasm and series of symptoms caused by hypervagotonia such as hyperhidrosis, salivation, slow heart rate, dizziness, etc. ${ }^{1,2}$. Modern pharmacological study has found that tropane alkaloids (scopolin, scopoletin) possess the function of antiinflammatory, anticancer, anti-HIV, relieving asthma and dieresis ${ }^{3-5}$. Chlorogenic acid, one kind of organic acids in $B$. tincture, has the function of restraining production of reactive oxygen, promoting bioavailability of nitric oxide, depressing oxidative stress, anticoagulation and reducing blood press ${ }^{6}$. In addition, some clinic reports have confirmed chlorogenic acid had antihypertensive effect for moderate hypertention ${ }^{7}$. At present, there has been no report on simultaneous determination in B. tincture in literature. Moreover, the quality control for $B$. tincture in Chinese Pharmacopoeia is restricted to determining Belladonna alkaloids by acid-base titration. In summary, its quality control is lack of specificity and could not monitor the determination of other active ingredients, existing large space for adulteration. the experiment established the method for the simultaneous determination of the five active ingredients (scopolin, chlorogenic acid, kaempferol-3-O- $\beta$-D (6-O- $\alpha$-L-rhamnogalactoside)-7-O- $\beta$-Dglucoside (KRG), scopoletin and rutin) in $B$. tincture. This method was stable with high precision, which could be used for the determination of the five ingredients and quality control of B. tincture.

\section{EXPERIMENTAL}

Shimadzu LC-20A type HPLC system (Shimadzu Co., Japan) was equipped with DGU-20A5 Degasser, LC-20AT Liquid Chromatograph, Sil-20AC HT Auto Sampler, SPDM20A Diode Array Detector and CTO-20AC Column Oven; Pa2251 electronic analytical balance (Sartorius Group, Germany); 5430R high speed centrifuge (USA, Eppendorf).

Standards of chlorogenic acid, scopoletin and rutin were supplied by National Institute for Food and Drug Control (NIFDC) (Beijing, China) with the batch number of 110753- 
200413, 110768-200504 and 100080-200306, respectively. Standards of scopolin and KRG were extracted and purified by ourselves (purity $>98 \%$ ). B. tincture was purchased from Luyin Pharmaceutical Co., LTD (Yantai, China). Methanol was of HPLC grade and other reagents used were of analytical grade. Deionized water was prepared using a Millipore water purification system.

HPLC conditions: The separation of scopolin, chlorogenic acid, KRG, scopoletin and rutin was carried out on an Agilent Kromesil 100-5C 18 (250 mm × 4.6 mm, $5 \mu \mathrm{m})$ column. The solvents used for HPLC separation were methanol (A) and $0.05 \%$ phosphoric acid (B) at a flow rate of $1.0 \mathrm{~mL} / \mathrm{min}$ with gradient elution (0-10 min, 3-15\% A; 5-60 min, 15-60\% A) and the analysis was monitored at $344 \mathrm{~nm}$ with the column temperature of $30^{\circ} \mathrm{C}$ and the injection volume was $10 \mu \mathrm{L}$.

Preparation of standard solution: Certain amounts of the 5 standard compounds (scopolin, chlorogenic acid, KRG, scopoletin and rutin) were dissolved with $50 \%$ methanol into volumetric flasks, respectively. Then measured $1 \mathrm{~mL}$ scopolin, $1 \mathrm{~mL}$ chlorogenic acid, $3.0 \mathrm{~mL}$ KRG, $1 \mathrm{~mL}$ scopoletin and $1 \mathrm{~mL}$ rutin standard solution, respectively, into a $10 \mathrm{~mL}$ volumetric flask and diluted to the concentrations of 32.6, 10, 30.9, 36.3 and $10.1 \mu \mathrm{g} / \mathrm{mL}$.

Preparation of sample solution: $B$. tincture was directly prepared for sample solution after high speed centrifugation.

\section{RESULTS AND DISCUSSION}

\section{Validation of the chromatographic method}

HPLC chromatograms of the five ingredients mixture (A) and Belladona tincture (B) are given in Fig. 1.

Linear range: Linear regression analysis for each component was performed by the external standard method. The above five compound solution was accurately injected 1, 2, 5, $10,15,20$ and $30 \mu \mathrm{L}$, respectively. The linearity of each compound was calculated by plotting the peak area (Y) vs. concentration (X) (Table-1). All the 5 components showed good linearities in wide concentration ranges.

TABLE-1

LINEAR REGRESSION EQUATION AND LINEAR RANGES

\begin{tabular}{llcc}
\hline \multicolumn{1}{c}{ Components } & $\begin{array}{c}\text { Regression } \\
\text { equation }\end{array}$ & $\begin{array}{c}\text { Correlation } \\
\text { coefficient } \\
\left(\mathrm{R}^{2}\right)\end{array}$ & $\begin{array}{c}\text { Linear range } \\
(\mu \mathrm{g})\end{array}$ \\
\hline Scopolin & $\mathrm{Y}=1652 \mathrm{X}-3995$ & 0.9999 & $0.0326-0.978$ \\
Chlorogenic acid & $\mathrm{Y}=1982 \mathrm{X}+3302$ & 0.9996 & $0.0100-0.300$ \\
KRG & $\mathrm{Y}=1468 \mathrm{X}+6857$ & 0.9996 & $0.0319-0.957$ \\
Scopoletin & $\mathrm{Y}=3723 \mathrm{X}+18460$ & 0.9995 & $0.0363-1.089$ \\
Rutin & $\mathrm{Y}=1561 \mathrm{X}+2226$ & 0.9994 & $0.0101-0.303$ \\
\hline
\end{tabular}

Precision: The standard mixture solution of scopolin, chlorogenic acid, KRG, scopoletin and rutin was injected into HPLC six times continuously and the area of each peak was used for the calculation of precision. The results showed that relative stand deviation (RSD) of peak area of each standard was $0.9,1.0,1.3,1.3$ and $1.9 \%$, respectively.

Stability: For stability test, the same sample solution was analyzed at designated time points in $24 \mathrm{~h}$. The results showed that RSDs of peak area were 1.2, 1.5, 1.7, 1.8 and $1.8 \%$ and found to be stable for the experiment.

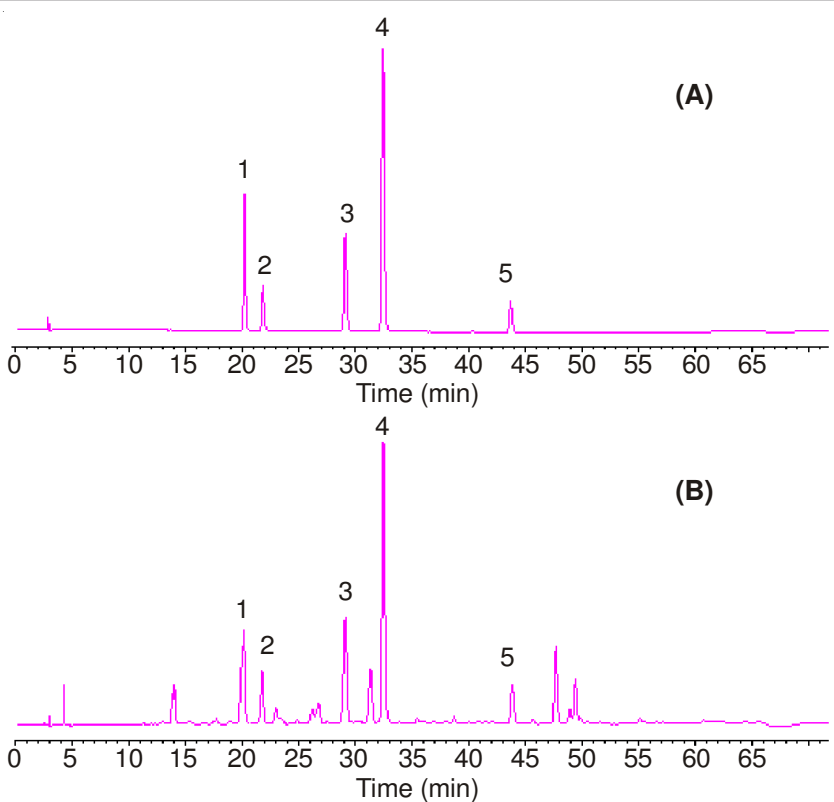

Fig. 1. HPLC chromatograms of the five ingredients mixture (A) and Belladona tincture (B). 1: scopolin; 2: chlorogenic acid; 3: KRG; 4: scopoletin; 5: rutin

Repeatability: Repeatability was carried out using six samples solution after the same treatment procedure. The results showed that RSD of each peak area was 1.1, 1.7, 1.9, 1.5 and $1.8 \%$, respectively.

Recovery test: The sample with known targeted contents was spiked with certain amounts of the 5 standards. Then the spiked sample was processed in accordance with the established method for the HPLC detection. The average recoveries for scopolin, chlorogenic acid, KRG, scopoletin and rutin determined were 99.59, 96.92, 96.41, 96.46 and 97.45\% (Table-2).

Application of HPLC method for quantitation studies: The experimental determination of three samples of different batches of the same manufacturer by the above method. The contents of the 5 components were showed in Table-3.

Optimization of HPLC separation conditions: In order to get a separation with better resolution of targeted components with shorter analytical time, we compared with three different column temperatures: 25,30 and $35^{\circ} \mathrm{C}$, then we found that the higher temperature, the shorter retention time with almost the same resolution, so we chose $30^{\circ} \mathrm{C}$ finally for protecting the lifespan of the column. In the meantime, we compared the efficiency of different elution solvents, namely methanolphosphoric acid and acetonitrile-phosphoric acid solution. The results indicated that the former was slightly better.

Six chromatographic columns of the same specification were compared in the experiment: Agilent Zorbax SB-C 18 , Agilent Kromasil 100-5C $\mathrm{C}_{18}$, Agilent Extend- $\mathrm{C}_{18}$, Phenomenex Luna- $\mathrm{C}_{18}$, Agilent Eclipse XDB- $\mathrm{C}_{18}$ and Welch Material XB$\mathrm{C}_{18}$, according to the effect of separation, Agilent Kromasil $100-5 \mathrm{C}_{18}(250 \mathrm{~mm} \times 4.6 \mathrm{~mm}, 5 \mu \mathrm{m})$ was used for the further research of the methodology.

\section{Conclusion}

In this study, 5 active components were quantitatively determined in $B$. tincture. To our knowledge, this new HPLC 


\begin{tabular}{|c|c|c|c|c|c|c|}
\hline \multicolumn{7}{|c|}{$\begin{array}{c}\text { TABLE-2 } \\
\text { RECOVERY RESULTS OF THE FIVE ACTIVE INGREDIENTS }\end{array}$} \\
\hline Component & $\begin{array}{c}\text { Contents in } \\
\text { samples (mg) }\end{array}$ & $\begin{array}{l}\text { Added } \\
(\mathrm{mg})\end{array}$ & $\begin{array}{c}\text { Found } \\
(\mathrm{mg})\end{array}$ & $\begin{array}{c}\text { Recovery } \\
(\%)\end{array}$ & $\begin{array}{c}\text { Mean } \\
(\%)\end{array}$ & $\begin{array}{c}\mathrm{RSD} \\
(\%)\end{array}$ \\
\hline \multirow{6}{*}{ Scopolin } & 0.0290 & 0.0326 & 0.0600 & 95.09 & \multirow{6}{*}{96.59} & \multirow{6}{*}{1.9} \\
\hline & 0.0290 & 0.0326 & 0.0617 & 100.29 & & \\
\hline & 0.0290 & 0.0326 & 0.0604 & 96.43 & & \\
\hline & 0.0290 & 0.0326 & 0.0602 & 95.69 & & \\
\hline & 0.0290 & 0.0326 & 0.0604 & 96.17 & & \\
\hline & 0.0290 & 0.0326 & 0.0602 & 95.85 & & \\
\hline \multirow{6}{*}{ Chlorogenic acid } & 0.0090 & 0.0100 & 0.0184 & 93.91 & \multirow{6}{*}{96.92} & \multirow{6}{*}{1.9} \\
\hline & 0.0090 & 0.0100 & 0.0189 & 99.01 & & \\
\hline & 0.0090 & 0.0100 & 0.0189 & 98.52 & & \\
\hline & 0.0090 & 0.0100 & 0.0187 & 96.92 & & \\
\hline & 0.0090 & 0.0100 & 0.0187 & 97.47 & & \\
\hline & 0.0090 & 0.0100 & 0.0186 & 95.71 & & \\
\hline \multirow{6}{*}{ KRG } & 0.0300 & 0.0319 & 0.0608 & 96.41 & \multirow{6}{*}{96.41} & \multirow{6}{*}{1.5} \\
\hline & 0.0300 & 0.0319 & 0.0616 & 98.94 & & \\
\hline & 0.0300 & 0.0319 & 0.0610 & 97.29 & & \\
\hline & 0.0300 & 0.0319 & 0.0604 & 95.28 & & \\
\hline & 0.0300 & 0.0319 & 0.0605 & 95.50 & & \\
\hline & 0.0300 & 0.0319 & 0.0603 & 95.06 & & \\
\hline \multirow{6}{*}{ Scopoletin } & 0.0250 & 0.0363 & 0.0600 & 96.49 & \multirow{6}{*}{96.46} & \multirow{6}{*}{2.2} \\
\hline & 0.0250 & 0.0363 & 0.0614 & 100.34 & & \\
\hline & 0.0250 & 0.0363 & 0.0601 & 96.82 & & \\
\hline & 0.0250 & 0.0363 & 0.0596 & 95.32 & & \\
\hline & 0.0250 & 0.0363 & 0.0596 & 95.22 & & \\
\hline & 0.0250 & 0.0363 & 0.0593 & 94.56 & & \\
\hline \multirow{6}{*}{ Rutin } & 0.0090 & 0.0101 & 0.0188 & 96.61 & \multirow{6}{*}{97.45} & \multirow{6}{*}{1.9} \\
\hline & 0.0090 & 0.0101 & 0.0190 & 99.30 & & \\
\hline & 0.0090 & 0.0101 & 0.0191 & 100.16 & & \\
\hline & 0.0090 & 0.0101 & 0.0187 & 95.94 & & \\
\hline & 0.0090 & 0.0101 & 0.0186 & 95.51 & & \\
\hline & 0.0090 & 0.0101 & 0.0188 & 97.18 & & \\
\hline
\end{tabular}

TABLE-3

CONTENTS DETERMINATION OF THE FIVE ACTIVE INGREDIENTS IN 3 BATCHES

\begin{tabular}{|c|c|c|c|c|c|}
\hline Batch number & Scopolin (mg/mL) & chlorogenic acid $(\mathrm{mg} / \mathrm{mL})$ & $\mathrm{KRG}(\mathrm{mg} / \mathrm{mL})$ & Scopoletin (mg/mL) & Rutin (mg/mL) \\
\hline 121409 & 0.0284 & 0.0100 & 0.0303 & 0.0253 & 0.0098 \\
\hline 121410 & 0.0293 & 0.0106 & 0.0316 & 0.0262 & 0.0104 \\
\hline 121411 & 0.0286 & 0.0102 & 0.0305 & 0.0254 & 0.0100 \\
\hline
\end{tabular}

method shortened the analysis time compared with former ones. On the whole, this developed method was simple, accurate for the determination of scopolin, chlorogenic acid, KRG, scopoletin and rutin simultaneously and reliable for the quality control and further efficacy study of $B$. tincture in clinic.

\section{REFERENCES}

1. State Pharmacopeia Committee of China, Chinese Pharmacopoeia, 2010 version, Part I, p. 1222 (2010).
2. L.T. Yang and S.L. Li, J. Appl. Clin. Pediatrics, 22, 1014 (2007).

3. L. He, S.L. Yang, D.S. Wu, T. Cui, D. Wei and Z.T. Ding, China J. Chin. Mater. Med., 37, 811 (2012).

4. S.Y. Zhang, L. Meng, W.Y. Gao, N.N. Song, W. Jia and H.Q. Duan, China J. Chin. Mater. Med., 30, 410 (2005).

5. X.H. Yang, J.H. Cui and A.W. Ding, J. Sichuan TCM, 24, 17 (2006).

6. A. Suzuki, N. Yamamoto, H. Jokura, M. Yamamoto, A. Fujii, I. Tokimitsu and I. Saito, J. Hyperten, 24, 1065 (2006).

7. T. Watanabe, Y. Arai, Y. Mitsui, T. Kusaura, W. Okawa, Y. Kajihara and I. Saito, Clin. Exp. Hypertens, 28, 439 (2006). 